Revue d'histoire de l'Amérique française

REVUE D.HISTOIRE DE L'AMÉRIQUE FRANÇAISE

\title{
La pensée économique de L'Action française (1917-1928)
}

\section{Jean-Claude Dupuis}

Volume 47, numéro 2, automne 1993

URI : https://id.erudit.org/iderudit/305217ar

DOI : https://doi.org/10.7202/305217ar

Aller au sommaire du numéro

Éditeur(s)

Institut d'histoire de l'Amérique française

ISSN

0035-2357 (imprimé)

1492-1383 (numérique)

Découvrir la revue

Citer cet article

Dupuis, J.-C. (1993). La pensée économique de L’Action française (1917-1928). Revue d'histoire de l'Amérique française, 47(2), 193-219.

https://doi.org/10.7202/305217ar

\section{Résumé de l'article}

La Ligue d'Action française a élaboré un plan de reconquête économique qui reposait sur l'organisation des ressources en capital et la réforme de l'éducation. Elle préconisait un rôle accru pour l'État, le contrôle des investissements étrangers et un développement équilibré des secteurs agricole et industriel. Elle était en faveur de la croissance de la richesse matérielle des Canadiens français mais tout en voulant préserver leur vocation messianique à nature spirituelle. C'est pourquoi elle proposait un modèle de développement qui rejetait à la fois le socialisme et le capitalisme sauvage. 


\title{
LA PENSÉE ÉCONOMIQUE DE L'ACTION FRANÇAISE (1917-1928)
}

\author{
JEAN-CLAUDE DUPUIS \\ Département d'histoire \\ Université Laval
}

\section{RÉSUMÉ}

La Ligue d'Action française a élaboré un plan de reconquête économique qui reposait sur l'organisation des ressources en capital et la réforme de l'éducation. Elle préconisait un rôle accru pour l'État, le contrôle des investissements étrangers et un développement équilibré des secteurs agricole et industriel. Elle était en faveur de la croissance de la richesse matérielle des Canadiens français mais tout en voulant préserver leur vocation messianique à nature spirituelle. C'est pourquoi elle proposait un modèle de développement qui rejetait à la fois le socialisme et le capitalisme sauvage.

\section{ABSTRACT}

The Ligue d'Action française drafted an economic reconquest plan that rested upon the organization of capital and educational reform. It advocated an increased role for the state, control of foreign investment and the balanced development of agriculture and industry. It favoured the growth of French Canadians' material wealth while preserving their identity and religious structure. That's why it privileged a model of development that rejected both socialism and unbridled capitalism.

L'Action française était sans doute la plus importante revue intellectuelle au Québec dans les années 1920. Elle fut, avec l'École Sociale Populaire, au cœur de la définition et de la diffusion de l'idéologie que l'on appelle habituellement «clérico-nationaliste», mais que nous préférons désigner sous le terme de traditionaliste ${ }^{2}$. Cette revue,

1. Le présent article résume une partie de Nationalisme et catholicisme. L'Action française de Montréal (1917-1928), mémoire de maîtrise (histoire), Université de Montréal, 1992, 329 p. Cette recherche a bénéficié d'une bourse du Fonds FCAR. Je remercie mon directeur, Pierre Trépanier, pour ses précieux conseils.

2. L'expression "clérico-nationaliste» est d'usage courant depuis que Pierre Elliott Trudeau l'a employée dans La grève de l'amiante (Montréal, Éditions de Cité libre, 1956), 430 p.; mais ce terme désigne mal l'idéologie en question et possède une connotation péjorative. Il serait plus juste de parler des traditionalistes comme le fait la sociologie française, car l'essence de cette école de pensée est la préservation de la continuité des traditions religieuses

RHAF, vol. 47, no 2, automne 1993 
dirigée par Lionel Groulx à partir de 1918, voulait doter les Canadiens français d'une «doctrine» qui orienterait tous les aspects de leur vie nationale: la religion, l'éducation, la culture, la politique et l'économie. La Ligue d'Action française chercha donc à développer une pensée nationale intégrale apte à résoudre les problèmes qui se posaient à ses compatriotes et, au premier chef, le problème de l'infériorité économique des Canadiens français. «La question nationale est une question économique», écrivait Édouard Montpetit dans l'article principal du premier numéro de la revue ${ }^{3}$. Dès le départ, L'Action française annonçait que son nationalisme serait d'abord un nationalisme économique.

Jusqu'à présent, les études qui ont porté sur L'Action française ont généralement sous-estimé l'importance de la question économique pour la revue. Sa pensée politique, et notamment la célèbre enquête de 1922 qui envisageait la formation d'un État français indépendant, a davantage retenu l'attention des chercheurs. Pourtant, la question économique dépassait largement la question constitutionnelle dans l'ordre des préoccupations de L'Action française. La plupart des études consacrées à la pensée économique de cette revue la rattachent injustement à la doctrine de l' «agriculturisme». Même si l'agriculture occupait une très grande place dans la pensée économique de L'Action française, celle-ci ne peut pas être qualifiée d'agriculturiste, du moins pas au sens que Michel Brunet donnait à ce terme ${ }^{4}$. D'ailleurs, les récents progrès historiographiques, qui semblent rejeter la théorie de l'agriculturisme, rendent nécessaire une relecture de la pensée économique de cette revue ${ }^{5}$. Les études antérieures furent réalisées dans

et nationales. Voir Pierre Barral, Les agrariens français de Méline à Pisani (Paris, Presses de la Fondation nationale des sciences politiques, 1968), 385 p. On pourrait aussi la qualifier tout simplement de catholique car la doctrine sociale de l'Église est, en définitive, la référence principale de cette école de pensée. Le terme clérico-nationaliste laisse entendre que le cléricalisme et le nationalisme sont au cœur de cette doctrine, ce qui n'est pas tout à fait exact. En fait, c'est la doctrine théologique, philosophique et sociale de l'Église catholique qui constitue le fondement de l'idéologie que nous voulons nommer. Certes, sur le plan pratique, le clergé et la nation occupent une grande place dans la pensée catholique, mais ils n'en constituent pas l'essence. Toutefois, puisque le terme catholique est large et qu'il peut porter à confusion, nous utiliserons dans cet article le terme traditionaliste pour désigner l'idéologie à laquelle adhérait L'Action française.

3. Édouard Montpetit, «Vers la supériorité», L'Action française (désormais $A F$ ) (janvier 1917): 1-7.

4. Michel Brunet, «Trois dominantes de la pensée canadienne-française: l'agriculturisme, l'anti-étatisme et le messianisme», La présence anglaise et les Canadiens (Montréal, Beauchemin, 1968), 113-166.

5. Dans un livre de synthèse qui reflète bien l'état actuel de l'historiographie, Nive Voisine ne fait aucune référence à la théorie de l'agriculturisme lorsqu'il décrit l'attitude de l'Église québécoise de la fin du XIX ${ }^{\mathrm{e}}$ siècle devant l'industrialisation et l'urbanisation. Philippe Sylvain et Nive Voisine, Histoire du catholicisme québécois. Réveil et consolidation (18401898) (Montréal, Boréal, 1991), 423-426. 
les années 1960 et 1970, alors que la thèse de la «Grande Noirceun", aujourd'hui délaissée par la plupart des historiens ${ }^{6}$, dominait encore l'historiographie québécoise. Nous étudierons donc la pensée économique de L'Action française à la lumière de cette révision historiographique.

Nous tenterons de démontrer que L'Action française était en faveur de l'industrialisation et de la modernisation économique du Québec. Elle y voyait même la condition sine qua non de la survie nationale des Canadiens français et de la réalisation de leur vocation spirituelle en Amérique du Nord. Toutefois, elle s'opposait fermement à la politique économique du gouvernement Taschereau, une politique de développement industriel rapide axée sur les investissements américains, les concessions massives de richesses naturelles, la concentration urbaine et le sacrifice conscient de l'agriculture à l'industrie $^{7}$. Elle craignait surtout que l'impulsion étrangère du mouvement d'industrialisation et d'urbanisation n'entraîne une acculturation des Canadiens français, une sorte d'américanisation par l'intérieur. L'Action française acceptait le progrès économique et technique, mais elle dénonçait le matérialisme philosophique qui y était souvent associé et qu'elle assimilait aux valeurs de la civilisation anglosaxonne. La revue préconisait un modèle alternatif de développement économique et social mieux adapté, selon elle, au «génie national» des Canadiens français. Ce modèle de développement visait, en définitive, à construire une société chrétienne de l'ère industrielle, c'est-à-dire une société moderne sur le plan technique, mais reposant sur les principes moraux de la doctrine sociale de l'Église plutôt que sur les règles du libéralisme économique. Ainsi, L'Action française subordonnait son programme économique, comme l'ensemble de son projet de société, à des préoccupations d'ordre spirituel. La revue proposait de développer une économie axée sur la décentralisation régionale, les petites et moyennes entreprises, le maintien de l'équilibre entre la croissance des secteurs industriel et agricole, le contrôle national des ressources naturelles, l'amélioration des lois sociales et l'intervention supplétive de l'État. Son programme de libération économique reposait sur deux principes fondamentaux: les progrès de l'instruction et la canalisation de l'épargne canadienne-française. L'objectif de ce programme était de relever le défi de la modernisation économique

6. Voir Fernande Roy, Progrès, harmonie, liberté. Le libéralisme des milieux d'affaires francophones à Montréal au tournant du siècle (Montréal, Boréal, 1988), 300 p.; Claude Couture, Le mythe de la modernisation du Québec, des années 1930 à la Révolution tranquille (Montréal, Méridien, 1991), $152 \mathrm{p}$.

7. Voir Bernard L. Vigod, Quebec Before Duplessis. The Political Career of LouisAlexandre Taschereau (Montréal et Kingston, McGill-Queen's University Press, 1986), 420 p. 
nord-américaine dans le respect des traditions culturelles et religieuses du Canada français.

Après avoir fait un bilan critique de l'historiographie, nous verrons en quoi la doctrine économique de L'Action française se démarquait du libéralisme alors dominant, et se rattachait plutôt à une autre famille de pensée économique qui rejetait à la fois le capitalisme sauvage et le socialisme. Nous présenterons ensuite quelques réflexions sur l'inadéquation d'une vision binaire des idéologies pour la compréhension de l'histoire intellectuelle du Québec de la première moitié du $\mathrm{XX}^{\mathrm{e}}$ siècle, vision binaire dont découle justement la méprise au sujet de l'agriculturisme.

\section{BILAN HISTORIOGRAPHIQUE}

Plusieurs études ont été consacrées à L'Action française, mais la seule qui aborde directement notre propos est la thèse de doctorat de Susan Mann-Trofimenkoff ${ }^{8}$. Sa présentation du fonctionnement interne de la Ligue d'Action française est excellente mais son analyse de l'idéologie de la revue laisse à désirer, surtout en ce qui a trait à la pensée économique. Trofimenkoff s'étonne que la question économique ait pu émerger dans une revue aussi «moralisatrice» que L'Action française. Selon elle, les trois caractéristiques de sa pensée

8. Susan Mann-Trofimenkoff, L'Action française: l'appel de la race, thèse de doctorat (histoire), Université Laval, 1970, 429 p.; thèse publiée sous le titre Action française: French Canadian Nationalism in the Twenties (Toronto, University of Toronto Press, 1975), $157 \mathrm{p}$. Autres études à signaler: Gérald Adélard Fortin, An Analysis of the Ideology of a French Canadian Nationalist Magazine: a Contribution to the Sociology of Knowledge, thèse de doctorat (sociologie), Cornell University, 1954, 251 p., analyse quantitative des thèmes abordés par L'Action française et L'Action nationale qui montre le glissement progressif de la revue vers des positions de plus en plus libérales, mais cette thèse ne contient pas d'analyse qualitative des idées propagées par ces deux périodiques; Louis-Philippe Jolicoeur, L'Action française de Montréal (1917-1928), mémoire de maîtrise (histoire), Université de Montréal, 1959, 173 p., qui comporte un bon index mais qui ne compte, en réalité, qu'un résumé de 40 pages des principaux thèmes de la revue et qui se concentre presque entièrement sur l'enquête de 1922; Jean-Louis Roy, Maître chez nous, dix années d'Action française (1917-1927) (Montréal, Leméac, 1968), 75 p., une petite plaquette qui résume la doctrine de la revue en alignant plusieurs citations les unes à la suite des autres mais qui ne contient aucune analyse; Donald Smith, «L'Action française (1917-1921)», Fernand Dumont, dir., Les idéologies au Canada français (1900-1929) (Québec, Presses de l'Université Laval, 1974), 345-367, article qui traite essentiellement du statut social des auteurs qui écrivaient dans la revue et qui se limite, curieusement, aux quatre premières années de publication; Yves Lamarche, «Position sociale des intellectuels et nationalisme: le cas de L'Action française», Philosophie au Québec (Montréal, Bellarmin, 1976), 153-178, analyse de type sociologique qui ne traite pas en tant que tel de l'idéologie de la revue; Ginette Bear, L'Action française de Montréal, mémoire de maîtrise (études françaises), Université McGill, 1977,90 p., qui étudie le volet littéraire de la revue. À l'exception de la thèse de Susan MannTrofimenkoff, toutes ces études n'abordent que très peu la question de la pensée économique de L'Action française. 
économique sont: 1) une approche moralisatrice et doctrinaire de la question; 2) une méfiance envers le matérialisme implicite de toute entreprise économique; 3) l'idée que l'industrialisation comportait pour les Canadiens français plus de dangers que d'avantages. En accord avec Michel Brunet, elle classe L'Action française dans le courant de pensée agriculturiste. Cependant, elle ajoute une nuance importante en précisant que l'agriculturisme était, dans les années 1920, aussi répandu au Canada anglais qu'au Québec. Trofimenkoff, signale dans une note de bas de page, une remarque de FrançoisAlbert Angers qui affirme que L'Action française ne s'opposait pas à l'industrialisation en tant que telle mais plutôt à certaines conséquences de l'industrialisation', et elle constate également que cette revue semblait s'opposer davantage à l'urbanisation qu'à l'industrialisation. Mais cette historienne n'a pas approfondi les implications de la remarque de François-Albert Angers et conclut finalement que la pensée économique de L'Action française était rétrograde. D'ailleurs, le titre du chapitre qu'elle consacre à cette question montre que Trofimenkoff a perçu la pensée économique de L'Action française essentiellement comme un refus de la modernisation: The Industrial Tocsin! Or, comme nous le verrons plus loin, les trois caractéristiques dégagées par Trofimenkoff ne correspondent pas à la réalité du discours de L'Action française.

Mais le sujet que nous abordons dans cet article nous oblige à tenir compte d'un corpus historiographique beaucoup plus vaste que celui qui porte directement sur L'Action française. En fait, notre étude implique une certaine révision de l'ensemble de la production historiographique qui traite de l'idéologie traditionaliste (ou clérico-nationaliste), et qui présente cette idéologie sous un aspect essentiellement négatif, comme un simple refus de la modernisation. En réalité, l'école de pensée traditionaliste avait son propre projet de modernisation, mais un projet différent de celui préconisé par les tenants de l'idéologie libérale.

Dans les années 1960 et 1970, de nombreuses études ont traité de la pensée économique des traditionalistes dans le cadre du débat sur les causes de l'infériorité économique des Canadiens français ${ }^{10}$. La question était alors de savoir si le nationalisme canadien-français

9. François-Albert Angers, «L'industrialisation et la pensée nationaliste traditionnelle», Robert Comeau, dir., Économie québécoise (Montréal, Presses de l'Université du Québec, 1969), 417-432.

10. Pour un excellent résumé de ce débat, voir René Durocher et Paul-André Linteau, Le retard du Québec et l'infériorité économique des Canadiens français (Montréal, Boréal Express, 1971), $127 \mathrm{p}$. 
traditionnel s'était opposé à l'industrialisation et si cette opposition avait eu un impact réel. Le débat tournait autour de la théorie de l'agriculturisme qui était défini comme «un refus de l'âge industriel contemporain qui s'inspire d'une conception statique de la sociétét ${ }^{11}$ ». François-Albert Angers était l'un des rares auteurs à contester le concept même d'agriculturisme ${ }^{12}$. La plupart des autres chercheurs admettaient l'existence d'une dominante agriculturiste dans la pensée nationaliste traditionnelle, tout en variant d'opinion sur l'importance qu'il fallait lui accorder. Michel Brunet reconnaissait lui-même que le programme économique de L'Action française était «audacieux pour l'époque ${ }^{13}$ ». Selon lui, il y avait parmi les traditionalistes quelques esprits «plus lucides» qui acceptaient le principe de la modernisation économique du Québec. D'autres chercheurs ont également nuancé l'importance de l'agriculturisme dans la pensée traditionaliste. Roger Bédard et Peter Southam ont présenté Édouard Montpetit comme un libéral aux idées avant-gardistes, «égaré» parmi les traditionalistes ${ }^{14}$. Southam signale aussi qu'Olivar Asselin s'opposait à un retour à la terre qui compromettrait l'avenir des Canadiens français dans l'industrie $^{15}$. Pierre Harvey a écrit que, pour Esdras Minville, l'agriculturisme était, dans le contexte de la crise des années 1930, une question d'opportunité et non pas une «doctrine permanente ${ }^{16}$ ». Yves Saint-Germain a qualifié Joseph Versailles, Édouard Montpetit, Marie-Victorin, Olivar Asselin et les membres de l'Association catholique de la jeunesse canadienne-française (ACJC) de «progressistes ambivalents» en matière économique ${ }^{17}$. Jacques Bélec perçoit égale-

11. Michel Brunet, op. cit., 119.

12. Voir François-Albert Angers, «Naissance de la pensée économique au Canada français», Revue d'histoire de l'Amérique française (désormais RHAF), 15,2 (septembre 1961): 204-229; «Nationalisme et vie économique», RHAF, 22,4 (mars 1969): 589-615; «L'industrialisation et la pensée nationaliste traditionnelle», Robert Comeau, dir., Économie québécoise, 417-432; "La pensée économique d'Esdras Minville», L'Action nationale (désormais $A N$ ), 65,9-10 (mai-juin 1976): 727-761. Signalons également un article qui s'attaque de façon très polémique à la thèse de Michel Brunet, Dominique Beaudin, «L'agriculturisme, margarine de l'histoire», $A N, 49,7$ (mars 1960): 506-530.

13. Michel Brunet, op. cit., 133.

14. Roger Bédard, «Edouard Montpetit, précurseur de la Révolution tranquille», $A N, 55,8$ (avril 1966): 899-928; Peter Southam, «La pensée sociale d'Édouard Montpetit», Fernand Dumont, dir., Les idéologies au Canada français (1930-1939) (Québec, Presses de l'Université Laval), 317-350.

15. Peter Southam, «La pensée économique d'Olivar Asselin», Robert Comeau, dir., Économie québécoise, 395-404.

16. Pierre Harvey, «Les idées économiques d'Esdras Minville des débuts à la maturité (1923-1936)», AN, 65,9-10 (mai-juin 1976): 627-642.

17. Yves Saint-Germain, «La société québécoise et la vie économique: quelques échos de la décennie de la grande ambivalence (1920-1929)», Robert Comeau, dir., Économie québécoise, 433-464. 
ment un conflit entre les tendances modernisante et agriculturiste de l'ACJC $^{18}$. Yves Roby affirme que les nationalistes des années 1920 voulaient réaliser l'indépendance économique du Canada français en s'appuyant sur l'agriculture qu'ils considéraient comme une «situation idéale», mais qu'ils préconisaient aussi, par réalisme, l'industrialisation du Québec ${ }^{19}$. William F. Ryan a sérieusement contesté la valeur de la théorie de l'agriculturisme en découvrant que le clergé des régions de Québec, Trois-Rivières et Chicoutimi avait, au tournant du siècle, encouragé activement l'industrialisation ${ }^{20}$. Cependant, Ryan n'a pas rejeté entièrement cette thèse. $\mathrm{Ne}$ s'appuyant que sur le célèbre article de Michel Brunet, il affirme que l'agriculturisme dominait effectivement la pensée de certains intellectuels urbains irréalistes, bien que cette doctrine n'ait pas eu d'impact réel sur l'industrialisation du Québec.

Tout en adhérant en principe à la théorie de la dominante agriculturiste, ces chercheurs ont relevé plusieurs éléments de «modernité» dans la pensée économique des traditionalistes. Ils expliquaient ce curieux phénomène par des «contradictions» ou des «ambivalences». Ces prétendues contradictions auraient opposé certains esprits plus «lucides», comme Montpetit ou Minville, à la tendance plus «rétrograde» de l'école de pensée traditionaliste. Toutefois, personne ne précise qui étaient les défenseurs de l'agriculturisme «pur et dur». Selon certains chercheurs, les fameuses contradictions pouvaient également se retrouver dans la pensée d'un même individu. Ainsi, AndréJ. Bélanger a perçu une «contradiction» dans le fait que Lionel Groulx idéalisait la vie rurale tout en préconisant une amélioration des techniques agricoles, «car les campagnes sont conçues (par Groulx), du moins en partie, comme des refuges contre l'envahissement de la modernité ${ }^{21}{ }^{1}$. Roger Payette ne comprend pas qu'Esdras Minville définisse l'autonomie économique nationale autrement que comme une simple appropriation de la grande industrie par les Canadiens français; il y voit une «contradiction» fondamentale de son nationalisme ${ }^{22}$.

18. Jacques Bélec, La pensée socio-économique de l'ACJC (1904-1935), mémoire de maîtrise (histoire), Université de Montréal, 1972, 146 p.

19. Yves Roby, Les Québécois et les investissements américains (1918-1929) (Québec, Presses de l'Université Laval, 1976), 81-118.

20. William F. Ryan, The Clergy and the Economic Growth in Quebec (1896-1914) (Québec, Presses de l'Université Laval, 1966), 348 p.

21. André-J. Bélanger, L'apolitisme des idéologies québécoises. Le grand tournant de 1934-36 (Québec, Presses de l'Université Laval, 1974), 231.

22. Roger Payette, Esdras Minville et le problème national des Canadiens français (1923-1956), mémoire de maîtrise (histoire), Université du Québec à Montréal, 1983, 53-56. 
Certes, il peut y avoir, du moins en apparence, des contradictions entre divers aspects du discours économique de L'Action française: modernisation économique et traditionalisme culturel, croissance parallèle des secteurs agricole et industriel, respect de la propriété privée et antilibéralisme, fonction subsidiaire de l'État et planification gouvernementale du développement. Toutefois, les divers éléments de ce discours perdent leur aspect contradictoire lorsque l'on tient compte du fait qu'ils s'intègrent dans une idéologie qui est aussi différente du libéralisme que du socialisme. Le principal défaut des études qui ont été consacrées à L'Action française est d'avoir classée cette revue, sous prétexte qu'elle était catholique et antisocialiste, parmi les défenseurs inconditionnels de l'entreprise privée, et d'avoir considéré sa pensée économique comme une simple variante, d'ailleurs «rétrograde», du libéralisme économique. Mais la pensée de L'Action française se situe dans un tout autre cadre idéologique, aussi distinct du libéralisme que du socialisme mais aussi répandu que ces deux derniers, une idéologie que nous appellerons, faute de mieux, la doctrine du nationalisme économique.

\section{ÉDOUARD MONTPETIT: ÉPARGNE ET INSTRUCTION}

Édouard Montpetit et Esdras Minville étaient les principaux économistes de L'Action française. Lionel Groulx traitait rarement de cette question sauf pour la situer par rapport à l'ensemble de la question nationale ${ }^{23}$. On peut distinguer deux périodes dans le discours économique de la revue. La première (1917-1923) est dominée par la pensée d'Édouard Montpetit, tandis que la seconde (1924-1928) est marquée par l'influence d'Esdras Minville. Il n'y a pas vraiment de

23. Plusieurs chercheurs, dont Susan Mann-Trofimenkoff, ont commis l'erreur d'assimiler entièrement la pensée de L'Action française à celle de Lionel Groulx. Celui-ci assurait la cohésion doctrinale de la revue, mais il n'imposait pas sa vision des choses sur tous les sujets. En matière économiqué, la doctrine de L'Action française était définie par Montpetit et, plus encore, par Minville; en matière religieuse, c'est la pensée de Mgr L.-A. Paquet et, dans une moindre mesure, celle du Père J.-M. Rodrigue Villeneuve qui prédominaient; en matière pédagogique, la revue s'appuyait sur les réflexions de l'abbé Georges Courchesne, de Mgr F.-X. Ross et de C.-J. Magnan. Lionel Groulx exerça davantage d'influence sur la définition du «nationalisme intégral» de L'Action française. Il ne faut jamais oublier que Groulx était d'abord un historien et un homme de lettres. On ne peut guère parler de sa «pensée économique» comme le fait Jean-Pierre Gaboury dans Le nationalisme de Lionel Groulx (Ottawa, Éditions de l'Université d'Ottawa, 1970), 161-185, ni de sa «pensée théologique» comme le fait Norman F. Cornett dans un récent article: «Lionel Groulx's Synthesis of the Universal and the Particular», ARC: The Journal of the Religious Studies (Universite McGill), 19 (1991): 87-105. Groulx n'était ni un économiste ni un théologien. À notre avis, les chercheurs s'épuisent inutilement à analyser sa pensée économique ou théologique (notez que nous ne disons pas religieuse); mieux vaut étudier directement les auteurs qui ont façonné les idées de Groulx sur ces questions, comme Esdras Minville ou Mgr Paquet. 
contradiction entre les deux périodes, mais il y a une accentuation des principes du nationalisme économique. Tout en rejetant la doctrine absolue du «laisser-faire», la pensée de Montpetit s'inscrivait malgré tout dans le sillage du libéralisme économique. Mais avec Esdras Minville, L'Action française s'éloigne davantage du modèle de développement libéral nord-américain et conçoit un projet de société plus conforme, selon elle, à la doctrine sociale de l'Église et aux traditions canadiennes-françaises.

Les thèmes centraux de la pensée économique d'Édouard Montpetit étaient la nécessité de développer l'instruction et le besoin de canaliser l'épargne canadienne-française par la pratique du «patriotisme des affaires». Précisant d'abord que la richesse matérielle est un moyen d'élever le Canada français dans l'ordre moral et intellectuel ${ }^{24}$, il écrivit en 1918 et 1919 deux articles qui jetaient les bases de la pensée économique de L'Action française ${ }^{25}$.

Montpetit se démarque immédiatement du libéralisme classique en dénonçant la doctrine du «laisser-faire» et en se définissant comme un «catholique social libéral ${ }^{26}$ ». Il préconise une certaine intervention de l'État dans les affaires économiques. Selon lui, il faut planifier et coordonner le développement économique en temps de paix comme en temps de guerre ${ }^{27}$. L'État devrait inventorier les ressources naturelles du Québec pour rationaliser leur exploitation et favoriser leur conservation, surtout dans le domaine forestier. Son rôle est de «stimuler l'industrie manufacturière par une politique qui soutienne les énergies mais sans se substituer à elles», car l'initiative privée doit demeurer le moteur du développement économique. Dans ce but,

24. Édouard Montpetit, «Vers la supériorité», $A F$ (janvier 1917): 2.

25. Édouard Montpetit, «Nos forces économiques», $A F$ (novembre 1918): 482-509; «Errol Bouchette et l'indépendance économique des Canadiens français», $A F$ (janvier 1919): 521.

26. La doctrine du catholicisme social fut élaborée en France par Albert de Mun et René de La Tour du Pin entre 1870 et 1890 . Elle visait à améliorer les conditions de vie de la classe ouvrière par l'intervention de l'État et l'action syndicale, mais tout en respectant la propriété privée et en maintenant le principe d'une société inégalitaire. Le catholicisme social s'opposait à la fois au capitalisme sauvage et au socialisme athée. Il devint en quelque sorte la doctrine officielle de l'Eglise avec la publication de Rerum novarum en 1891. L'expression «catholique social libéral» est plutôt ambiguë. Montpetit voulait probablement dire que, même s'il se préoccupait de la question sociale, il ne remettait pas en cause le principe du libre jeu des forces économiques. Les véritables catholiques sociaux, comme Arthur Saint-Pierre et Esdras Minville, étaient plus interventionnistes que Montpetit, plus favorables à une forme de régulation économique, par l'État ou par la corporation. Voir Jean-Claude Dupuis, «Réformisme et catholicisme. La pensée sociale d'Arthur Saint-Pierre», Bulletin du regroupement des chercheurs-chercheures en histoire des travailleurs et travailleuses du Québec, 17,1 (hiver 1991): 25-61.

27. Montpetit se laisse sans doute entraîner ici par sa prose car la planification économique du temps de guerre est très dirigiste. Or, comme nous le verrons plus loin, il réclamait de l'État une «coordination» plutôt qu'une «direction» économique. 
Montpetit suggère de créer un ministère provincial de l'industrie, du travail et de la statistique.

S'inspirant d'Errol Bouchette ${ }^{28}$, Montpetit préconise une politique économique «organisatrice», une expression qui rend mieux sa pensée que celle de politique économique «interventionniste». Il constate que la concentration industrielle est l'aboutissement nécessaire de l'évolution économique, mais qu'elle devient nuisible lorsqu'elle prend la forme de trusts qui neutralisent la concurrence et qui s'approprient le marché. Il serait toutefois possible, dit-il, d'obtenir les avantages de la concentration, tout en évitant ses inconvénients, grâce à une politique industrielle méthodique qui tiendrait compte du «génie national» des Canadiens français. Nous retrouvons ici le lien que L'Action française établissait entre le développement économique et la question nationale; mais notons que le sens de l'expression «génie national» restait vague dans les pages de la revue.

La croissance économique du Canada français devait, dans l'esprit d'Édouard Montpetit, s'appuyer sur l'alliance de la science et de l'industrie. Il citait en exemple les vieilles universités britanniques qui avaient su intégrer la nouvelle discipline de la science commerciale. Montpetit réclamait la création d'une faculté des sciences à l'Université de Montréal et l'ouverture d'une section de génie chimique à l'École polytechnique. Il suggérait de diminuer la charge d'enseignement des professeurs pour leur permettre de faire de la recherche. À l'avenir, écrivait-il, les chefs d'entreprises ne pourront plus compter uniquement sur un apprentissage par la pratique des affaires, mais ils auront besoin d'une formation théorique comme celle dispensée par l'École des hautes études commerciales. Cependant, il ajoutait que la formation de la future élite commerciale canadienne-française devait reposer sur une solide culture générale. C'est pourquoi l'enseignement classique devait demeurer au cœur du système scolaire québécois.

Montpetit affirme que l'industrie moderne exige une organisation scientifique du travail. Il faut donc développer l'enseignement technique et l'apprentissage en usine. De plus, dit-il, le progrès des lois sociales et du syndicalisme renforce la productivité. Le taylorisme doit être appliqué avec modération. Montpetit approuve ainsi le réformisme ouvrier, mais dans l'optique du syndicalisme catholique.

Selon Édouard Montpetit, le problème économique est d'abord un problème industriel et commercial ${ }^{29}$, mais il ne néglige pas pour autant

28 Errol Bouchette, L'indépendance économique du Canada français (Arthabaska, La compagnie d'imprimerie d'Arthabaska, 1906), $334 \mathrm{p}$.

29. Fait significatif, Montpetit a consacré dans son article de 1918 seulement 2 pages sur 27 aux problèmes de l'agriculture. 
l'agriculture. Il considère que le sol est la plus sûre richesse des Canadiens français. La vie rurale qui prête, dit-il, à plus d'un développement poétique, fut historiquement le refuge de la nationalité. Cependant, Montpetit ajoute que l'agriculteur est désormais un homme d'affaires. Il faut donc diffuser l'enseignement agricole, créer des fermes modèles et former des agronomes qui répandront la pratique d'une agriculture scientifique. Toutefois, il ne faut pas considérer la modernisation agricole préconisée par Montpetit comme une sorte d' «agriculturisme éclairé» car, dans son esprit, l'agriculture devait servir de tremplin à l'essor industriel du Canada français. Le «bas de laine paysan» fournirait les capitaux nécessaires au développement d'une industrie nationale canadienne-française.

Nous voyons ici le lien que L'Action française établissait entre le développement agricole et le développement industriel. La clef de voûte de son programme de libération économique était la canalisation de l'épargne par la pratique de la solidarité nationale en affaires. La revue incitait fortement ses compatriotes à placer leurs avoirs dans des banques ou des compagnies d'assurances canadiennes-françaises et à développer des habitudes d' "achat chez nous». Mais encore fallait-il que cette épargne existe. Or les propriétaires terriens pouvaient disposer de plus d'épargne que les prolétaires urbains, surtout à une époque où la classe ouvrière vivait au seuil de la misère. C'est pourquoi la prospérité du monde rural était si importante aux yeux de L'Action française. Ce n'était pas là rêverie bucolique mais calcul financier. Il fallait dégager les capitaux nécessaires au développement d'une industrie canadienne-française, et ces capitaux proviendraient de la campagne plus que de la ville.

Certes, il y a dans L'Action française des passages qui reflètent un agriculturisme sentimental, une idéalisation de la vie champêtre. Mais il faut remarquer que ces passages ne se trouvent pas dans les textes qui traitent d'économie, et encore moins dans ceux qui traitent d'agriculture. Ces derniers sont écrits par des agronomes qui abordent la question d'un point de vue très technique ${ }^{30}$. Les allusions agriculturistes se retrouvent plutôt dans des textes qui traitent de littérature ou de morale ${ }^{31}$. Beaucoup d'historiens n'ont pas fait cette distinction, ce

30. Voir la série d'articles de l'agronome Charles Gagnier sur «Notre problème agricole» parue dans les numéros de février, mars et avril 1924.

31. Voir Irène Lepage, «L'éducation patriotique des enfants», $A F$ (février 1918): 56-60; Louis Dupire, «Les Rapaillages», $A F$ (juin 1919): 274-275 (critique du livre de Lionel Groulx); F. Charbonnier, «La campagne canadienne, croquis et leçons», $A F$ (avril 1925): 241-248 (critique du livre d'Adélard Dugré, S.J.). 
qui a causé une certaine confusion. Jean-Pierre Gaboury, par exemple, ne semble pas avoir saisi ce qu'il y avait de littéraire et d'historique dans les «envolées agriculturistes» du chanoine Groul ${ }^{32}$.

\section{L'ENQUÊTE DE 1921}

L'Action française publia en 1921 une enquête sur Le problème économique. Depuis Errol Bouchette, personne n'avait étudié cette question d'une manière aussi complète. À cette époque, Esdras Minville ne collaborait pas encore à la revue, de telle sorte que la doctrine qui se dégage de l'enquête demeure dans la ligne de pensée d'Édouard Montpetit. Les collaborateurs insistent sur l'instruction, la science, l'épargne, l'action organisatrice de l'État, le rejet du «laisserfaire» et l'importance de l'agriculture; mais ils ne se démarquent pas encore entièrement du libéralisme économique. Notons surtout qu'on n'y décèle aucune hostilité envers l'industrialisation, comme l'indique ce passage d'Édouard Montpetit: «Ce sera le mérite de l'industrialisme contemporain que d'avoir permis à l'homme de vivre un peu plus la vie de l'esprit et du cœur en s'assurant un peu mieux chaque jour la vie du $\operatorname{corps}^{33}$.»

Lionel Groulx et Antonio Perrault ont précisé les rapports que L'Action française établissait entre son programme de libération économique et les aspects national et religieux de sa doctrine.

Citant Georges Valois ${ }^{34}$, Groulx affirme que les Canadiens français ont besoin d'une «doctrine» pour orienter leur effort de libération économique ${ }^{35}$. Le directeur de L'Action française s' inquiète des transformations économiques qui bouleversent le monde et qui menacent l'avenir des Canadiens français. Il ne craint pas l'industrialisation en tant que telle mais plutôt le «culte des forces matérielles» qui l'accompagne. Cependant, ajoute Groulx, la guerre économique existe et la province de Québec possède trop de richesses pour ne pas attirer les grandes puissances financières. La question n'est plus de savoir si ces richesses seront exploitées ou non, mais plutôt si elles seront

32. Pierre Gaboury, op. cit., 175-176.

33. Édouard Montpetit, «L'indépendance économique des Canadiens français», $A F$ (janvier 1921): 7.

34. Georges Valois, L'Économie nouvelle. L'intelligence et la production (Paris, Nouvelle librairie nationale, 1925), 569 p. (première édition 1919). Notons que Lionel Groulx ne traite pas des aspects véritablement économiques de cet ouvrage, comme la critique de la théorie de l'offre et de la demande, les facteurs de production ou l'organisation corporative. Il ne semble avoir retenu que l'aspect moral du livre de Valois, ce qui montre à quel point Groulx se désintéressait de la science économique. Ce n'est que l'aspect national du problème économique qui le préoccupait.

35. Lionel Groulx, «Le problème économique», $A F$ (décembre 1920): 558-565. 
exploitées par les Canadiens français dans leur intérêt ou par des étrangers contre l'intérêt des Canadiens français.

Lionel Groulx ne rejetait pas le fait industriel mais cherchait à l'orienter dans le sens des valeurs catholiques et des traditions canadiennes-françaises. Pour ce faire, il proposait une doctrine qui ne se limitait pas au seul domaine économique mais qui impliquait le développement intégral d'une conscience nationale. La doctrine économique de L'Action française reposait sur quelque chose de plus vaste, sur une doctrine nationaliste intégrale impliquant une nouvelle conception de l'État provincial québécois. L'enquête de 1921 tendait ainsi le flambeau à l'enquête de 1922.

Le domaine national, écrit Groulx, le capital d'exploitation n'ont jamais eu pour nos gouvernants de nationalité parce que, pour eux, l'État n'en avait point. Eh bien, c'est cette incroyable erreur qui doit prendre fin. Il appartiendra à la jeune génération, si elle veut atteindre aux réalisations puissantes, de faire admettre que l'être ethnique de l'État québécois est fixé depuis longtemps et de façon irrévocable. [...] toutes les constitutions politiques depuis 1774, ont fait du Québec un État français qu'il faut reconnaître en théorie comme en fait. C'est cette vérité qu'il faut placer en haut pour qu'elle y gouverne chez nous dans l'ordre économique, comme on admet spontanément qu'elle doive gouverner les autres fonctions de notre vie ${ }^{36}$.

Lionel Groulx ne lie pas seulement le programme économique de L'Action française à des préoccupations d'ordre national mais aussi à des préoccupations d'ordre moral et spirituel. S'inspirant de Rerum novarum, il rejette la «théorie funeste du laisser-faire» de l'économie libérale. Groulx affirme qu'aucune activité humaine ne peut échapper aux lois souveraines de l'ordre moral et que les pouvoirs publics ont des obligations positives en matière économique. Il insiste sur le fait que le programme économique de L'Action française se rattache étroitement à la question sociale et qu'il dépasse même l'intérêt strictement national. Il ne servirait à rien, dit-il, que les Canadiens français soient les vainqueurs de la lutte économique s'ils devaient être les vaincus de la richesse, si leur nationalité en venait à subordonner le spirituel au matériel et à sortir de «l'ordre latin».

Nous voyons ici que la pensée économique de L'Action française ne visait pas seulement à créer une économie nationale mais qu'elle visait aussi, et même surtout, à créer un type d'économie conforme aux valeurs d'une société chrétienne. 
Antonio Perrault a développé davantage l'aspect moral du problème économique pour répondre à ceux qui craignaient que l'enrichissement des Canadiens français ne fausse leur vocation spirituelle en matérialisant leur culture ${ }^{37}$. Selon lui, le progrès matériel ne constitue pas nécessairement une menace pour le progrès moral et spirituel. Il suffit de ne pas poser de cloison entre la vie privée et la vie publique et de ramener à l'unité les divers éléments constitutifs du progrès. Ainsi, un commerçant ne peut pas demeurer un honnête chrétien dans sa vie privée et être dépourvu de tout principe moral en affaires. La doctrine libérale du bien commun par la somme des égoïsmes individuels est fausse, car si elle produit, du moins en apparence, un progrès matériel, elle provoque inévitablement une régression morale. Or le progrès social forme une unité indivisible, à la fois matérielle, culturelle et spirituelle. C'est pourquoi, affirme Perrault, les théories économiques ou politiques qui ne sont pas liées aux «données supérieures de l'âme» sont dangereuses. Mais L'Action française, dit-il, entend réunir les aspects matériel et moral du problème économique pour l'envisager dans toute son ampleur.

Perrault rappelle que l'Église ne condamne pas la richesse des biens extérieurs nécessaires à la prospérité matérielle des familles et des sociétés; par contre, elle condamne le matérialisme qui borne aux choses secondaires l'horizon de l'humanité. Cependant, il croit qu'il est possible de concilier le progrès matériel et le renoncement chrétien. De toute façon, la nation canadienne-française ne peut pas se permettre d'abandonner la richesse à ses rivaux anglo-saxons. «C'est pour sauvegarder son âme et ce qui en fait la valeur qu'elle doit vouloir conquérir les biens économiques ${ }^{38}$.» Il reconnaît à la «richesse jouissance», celle des petits travailleurs, une sorte de supériorité morale sur la «richesse puissance», celle des grands industriels et des grands financiers. Mais il conclut malgré tout que les Canadiens français ne peuvent pas dédaigner la «richesse puissance ${ }^{39}{ } \gg$.

Les textes de Groulx et de Perrault se démarquent du libéralisme économique en liant le progrès matériel et le progrès moral. En outre, ils marquent une profonde différence entre la pensée économique de L'Action française et la pensée d'Errol Bouchette élaborée vingt ans plus tôt.

37. Antonio Perrault, «L'aspect moral», $A F$ (février 1921): 66-87.

38. Ibid., 74.

39. Perrault tire cette distinction entre «richesse jouissance» et «richesse puissance» de l'œuvre de Charles Perrin, professeur à l'Université de Louvain et théoricien de l'économie sociale chrétienne. 
L'Action française présentait Errol Bouchette comme un maître à penser. La revue s'inspirait d'ailleurs largement du programme économique qu'il avait proposé. Mais en réalité, L'Action française ne retenait de cet auteur que sa politique de développement industriel et non pas l'ensemble de sa pensée. Sur les plans national et social, elle en divergeait profondément. Contrairement à L'Action française, le patriotisme d'Errol Bouchette était pancanadien. Il ne tarissait pas d'éloges sur l'empire britannique et préconisait une intégration des deux nationalités canadiennes. Alors que L'Action française adhérait au catholicisme social, Bouchette semblait attiré par le travaillisme britannique ${ }^{40}$. Errol Bouchette préconisait une «méthode» économique, mais cette expression signifiait seulement une politique de développement industriel. $\mathrm{La}$ «doctrine» dont parle Groulx déborde le domaine économique pour toucher aux fondements de l'identité nationale des Canadiens français. En ce sens, la portée de la pensée économique de L'Action française dépassait celle d'Errol Bouchette.

Après que Groulx et Perrault eurent défini les principes moraux qui devaient guider la doctrine économique de L'Action française, les autres collaborateurs ont dévoilé les aspects pratiques du programme. Georges Pelletier propose d'industrialiser la production agricole ${ }^{41}$ et Anatole Vanier réclame la mise sur pied d'écoles moyennes d'agriculture $^{42}$. Émile Miller suggère de créer un ministère provincial des ressources naturelles ${ }^{43}$; Léon Lorrain demande au gouvernement de développer une marine marchande nationale et un service de renseignements commerciaux ${ }^{44}$; Beaudry Leman vante la carrière de banquier ${ }^{45}$ et Omer Héroux explique comment les assurances et les mutualités peuvent servir d'accumulateurs de capitaux ${ }^{46}$. J.-E. Gendreau affirme que les peuples dominants de l'avenir seront ceux dont les laboratoires auront porté au plus haut point la production industrielle $\mathrm{e}^{47}$. Le Canada français, dit-il, doit devenir, à l'instar de la France, une «nation à structure scientifique». Il souhaite la fondation d'un Institut provincial de recherches pour développer, notamment, l'utilisation de l'énergie nucléaire. Henry Laureys, directeur des HEC, propose de

40. Errol Bouchette, op. cit., 8-28.

41. Georges Pelletier, «Notre industrie», $A F$ (juin 1921): 322-356.

42. Anatole Vanier, «Le facteur agricole», $A F$ (mai 1921): 258-273.

43. Émile Miller, «Notre avoir économique», $A F$ (avril 1921): 194-208.

44. Léon Lorrain, «Le commerce canadien-français», $A F$ (juillet 1921): 386-396.

45. Beaudry Leman, «Les institutions de crédit», $A F$ (août 1921): 450-464.

46. Omer Héroux, «Assurances et mutualités», $A F$ (octobre 1921): 578-584.

47. J.-E. Gendreau, «Le problème économique et l'enseignement scientifique supérieur», $A F$ (novembre 1921): 642-657. 
réformer le cours commercial moyen en s'inspirant du système scolaire belge ${ }^{48}$.

Les collaborateurs de L'Action française divergeaient d'opinion sur l'épineux problème du contrôle des richesses naturelles par les étrangers. Édouard Montpetit acceptait de laisser l'exploitation de ces richesses aux capitaux étrangers tant que les Canadiens français n'auraient pas les moyens de le faire eux-mêmes. Georges Pelletier proposait plutôt de les laisser en friche jusqu'au jour où ses compatriotes seraient en mesure de les développer ${ }^{49}$. Olivar Asselin dénonçait vigoureusement les concessions à long terme accordées par le gouvernement Taschereau, mais ne suggérait pas vraiment de solution de rechange si ce n'est de réduire la durée des baux et d'augmenter les redevances ${ }^{50}$.

L'Action française renonçait d'avance à résoudre ce problème par l'expropriation. En accord avec la doctrine sociale de l'Église, elle avait un grand respect pour la propriété privée qu'elle considérait de droit naturel. Ce droit ne pouvait être brimé que pour des raisons majeures. Ainsi, Georges Pelletier écrivait que, même s'il devenait indépendant, le Canada français devrait respecter les contrats conclus entre la province de Québec et les investisseurs étrangers. Cependant, la revue a publié en 1928 un article d'Olivar Asselin qui suggérait d'étatiser les exploitations hydro-électriques de la province ${ }^{51}$. Les nationalistes commençaient alors à répandre une idée qui deviendra leur leitmotiv dans les années 1930.

L'enquête de 1921 portait essentiellement sur le problème industriel; le thème de l'agriculture restait secondaire. Á cette époque, L'Action française concevait encore l'avenir économique du Québec dans une optique assez proche du libéralisme. Elle préconisait l'édification d'une économie nationale mais non une économie différente. Cependant, les textes de Lionel Groulx, d'Antonio Perrault et même, dans une certaine mesure, de Montpetit ouvraient la porte à une conception du développement économique radicalement différente du modèle libéral nord-américain, soit l'idée d'une économie fondée sur les principes du catholicisme social. Mais cette nouvelle conception ne

48. Henry Laureys, «Notre enseignement commercial et technique», $A F$ (septembre 1921): $514-545$.

49. Georges Pelletier, «Les obstacles économiques à l'indépendance du Canada français», $A F$ (août 1922): 66-82.

50. Olivar Asselin, «Les lacunes de notre organisation économique», $A F$ (mars 1921): 130-146.

51. Olivar Asselin, «L'industrie dans l'économie du Canada français», L'Action canadienne-française (désormais $A C F$ ) (septembre 1928): 175. 
s'exprimait encore qu'en termes philosophiques et moraux. Avec Esdras Minville, elle se traduira véritablement en doctrine économique.

\section{ESDRAS MINVILLE: LA RADICALISATION DU NATIONALISME ÉCONOMIQUE}

La pensée économique d'Esdras Minville visait essentiellement à maintenir un double équilibre, d'une part, entre le développement du centre et celui des zones périphériques et, d'autre part, entre les progrès de l'industrie et ceux de l'agriculture. Sa pensée était régionaliste plutôt qu'agriculturiste. Certes, il insistait beaucoup sur les problèmes de l'agriculture, mais il ne s'opposait pas au développement industriel. Il concevait toutefois ce développement dans une optique régionale et, surtout, nationale. Dans son esprit, l'économie régionale serait constituée d'une chaîne de petites et moyennes industries appuyée sur le secteur agricole, le seul que possédait vraiment les Canadiens français. L'agriculture devait servir de tremplin, et non de frein.

À cette époque, presque tous les économistes, même libéraux, accordaient une importance capitale au secteur agricole. N'oublions pas que, dans les années 1920, l'agriculture comptait encore pour près de 50\% du PNB canadien. A. J. de Bray, par exemple, écrivait: «L'agriculture occupera la première place, indéfiniment pourrait-on dire, parce que si l'évolution industrielle est plus rapide actuellement, c'est en raison de ce qu'elle est à ses débuts; elle devra ralentir et les courbes des progrès futurs de ces deux branches de l'activité ne tarderont pas à devenir parallèles ${ }^{52}$.» Montpetit affirmait également que l'agriculture ne devait pas être sacrifiée à l'industrie. Peu d'économistes prévoyaient alors que le secteur industriel absorberait presque complètement le monde rural, comme il le fit après la Seconde Guerre mondiale. D'ailleurs, la crise de 1929 sembla donner raison à ceux qui prétendaient que la croissance industrielle ne pouvait pas durer indéfiniment. C'est pourquoi l'originalité de la pensée d'Esdras Minville ne tient pas tant à la place qu'il réservait au secteur agricole qu'à l'importance qu'il accordait à la décentralisation économique et démographique. L'agriculture n'était qu'un moyen parmi d'autres de fonder une solide économie régionale, un moyen d'ailleurs adapté aux conditions techniques de son temps, mais un moyen qui n'était pas nécessairement appelé à durer éternellement. Dans les

52. A. J. de Bray, L'essor industriel et commercial du peuple canadien (Montréal, Librairie Beauchemin, 1912), 65. 
années 1930, alors que l'idée du «retour à la terre» était beaucoup plus à la mode que dans les années 1920, Minville se fera le défenseur de la «vocation industrielle» du Québec ${ }^{53}$.

Pour comprendre la pensée d'Esdras Minville et de L'Action française, il importe de faire une distinction entre le régime de la propriété privée et le libéralisme économique. Le principe de la propriété privée est fondé sur le droit naturel et s'accorde avec la doctrine sociale de l'Église. En outre, la philosophie thomiste reconnaît à l'État un rôle subsidiaire, c'est-à-dire un rôle de suppléance lorsque l'initiative privée, des individus ou des familles, est insuffisante à réaliser le bien commun. La propriété privée et la subsidiarité de l'État sont les principes de base de ce que l'on pourrait appeler une «économie chrétienne ${ }^{54} »$.

Le libéralisme économique est également fondé sur le droit de propriété, mais il ne rattache pas nécessairement ce droit à la loi naturelle. Il le justifie plutôt en vertu de la raison. Ainsi, la doctrine libérale affirme que le bien commun découle de l'affrontement des intérêts privés dans le cadre régulateur de la loi du marché (la «main invisible» d'Adam Smith ${ }^{55}$. De plus, le libéralisme économique rejette, du moins en théorie, toute intervention de l'État, sauf en ce qui a trait au maintien de l'ordre public, et plus particulièrement à la protection de la propriété privée. En schématisant, on pourrait dire que la loi du marché est à l'économie libérale ce que la loi de Dieu est à l'économie chrétienne, soit la justification idéologique du système économique.

En accord avec la doctrine sociale de l'Église, L'Action française respectait le principe de l'entreprise et de la propriété privées, mais elle ne croyait pas que la loi du marché pouvait à elle seule résoudre le problème de l'injustice sociale. Elle était antilibérale sans être socialiste. S'inspirant de Rerum novarum, la revue favorisait l'intervention de l'État en vue du bien commun, mais une intervention supplétive, c'est-à-dire qui se limite aux cas où l'initiative privée reste insuffisante. Ce type d'interventionnisme est différent de l'interventionnisme socialiste qui établit en principe que l'État est nécessairement supérieur aux institutions privées (famille, Église, entreprise) pour assumer certaines fonctions sociales.

53. Voir François-Albert Angers, «La pensée économique d'Esdras Minville», Robert Comeau, dir., Économie québécoise, 465-483.

54. Voir Émile Marmy, La communauté humaine selon l'esprit chrétien (documents) (Fribourg-Paris, Éditions Saint-Paul, 1949), 911 p.; J.-Y. Calvet et J. Perrin, Église et société économique. L'enseignement social des papes de Léon XIII à Pie XII (Paris, Aubier, 1959), $578 \mathrm{p}$.

55. Voir André Vachet, L'idéologie libérale (Paris, Anthropos, 1970), 511 p. 
Les deux premiers articles de Minville publiés dans L'Action française dénotent un antiaméricanisme beaucoup plus prononcé que tout ce que l'on avait pu lire jusqu'alors dans la revue ${ }^{56}$. Selon lui, les capitaux américains qui envahissent le Québec créent un «sur-État économique» capable de tenir en laisse l'État politique. Si le gouvernement, dit-il, aide avec autant de zèle la grande industrie étrangère alors qu'il fait si peu pour soutenir la petite industrie rurale (au sens d'industrie régionale liée à l'agriculture et non pas au sens d'exploitation agricole), c'est parce que la «force occulte de la puissance financière» est déjà en mesure d'imposer ses volontés au pouvoir public. Aux partisans du libéralisme économique qui prétendent qu'il ne faut pas mêler le patriotisme et les affaires, Minville réplique que les entrepreneurs canadiens-français se heurtent à l'hostilité de la «caste financière anglo-saxonne» qui, elle, n'hésite pas à manifester son «patriotisme d'affaires». Avec l'arrivée d'Esdras Minville au sein de la Ligue d'Action française, la dénonciation du «sur-État économique» deviendra un leitmotiv de la revue ${ }^{57}$. Ce thème n'avait jamais été abordé par Montpetit qui semblait moins défavorable aux investissements étrangers. Certes, Minville ne s'opposait pas à toute forme de capital étranger. Il reconnaissait qu'un jeune pays comme le Canada en avait besoin, mais à condition qu'il soit un allié et non un maître.

Esdras Minville était plus interventionniste qu'Édouard Montpetit. Dans l'enquête de 1927 qui résumait la doctrine de L'Action française, on peut lire un passage dont le ton s'éloigne de la timide politique «organisatrice» préconisée par Montpetit. Il y est question de "plan» plutôt que de simple «coordination».

Et qui dressera et fera exécuter le plan selon lequel ces industries surgiront du sol, pour le plus grand avantage de la population tout entière cette fois, et non pas seulement pour celui d'une poignée d'entrepreneurs étrangers comme c'est le cas, à l'heure actuelle, de la plupart des entreprises qui fleurissent sur notre sol? L'État! et oui, l'État! en toute vérité, l'État lui-même, puisqu'aussi bien son rôle consiste à stimuler l'initiative privée insuffisante, à remplacer l'initiative privée impuissante, à encourager l'initiative privée suffisante ${ }^{58}$.

Bien qu'ils n'aient pas exactement la même attitude devant le problème des investissements étrangers et la question de l'intervention

56. Esdras Minville, «Les Américains et nous», $A F$ (août 1923): 97-105; «Le capital étranger», $A F$ (juin 1924): 323-349.

57 «Mot d'ordre: secouons le joug», $A F$ (août 1923): 65.

58. «Industrie et commerce», $A F$ (octobre 1927): 212-213. Les articles de l'enquête de 1927 n'étaient pas signés mais on reconnaît, sinon la plume, du moins l'esprit de Minville. 
de l'État, il n'y a pas vraiment de rupture de pensée entre les deux économistes de L'Action française. Minville ne rejetait pas l'œuvre de son prédécesseur: il la complétait, la radicalisait et lui donnait plus de cohérence en l'intégrant davantage dans une doctrine nationale fondée sur les principes sociaux catholiques. Toutefois, comme l'a remarqué François-Albert Angers, Édouard Montpetit n'a jamais eu l'enthousiasme d'Esdras Minville pour l'idée de planification économique ${ }^{59}$.

Minville se démarquait aussi des autres collaborateurs de L'Action française lorsqu'il envisageait d'étendre l'action des coopératives à des organisations industrielles de petite et moyenne envergure. Olivar Asselin pensait que les coopératives ne pouvaient jouer un rôle qu'en matière agricole. L'Action française encourageait le développement des caisses populaires, mais aucun des collaborateurs de la revue ne semble avoir cru qu'elles pourraient devenir un jour pour les Canadiens français un puissant levier de libération économique. Sur ce point, les nationalistes se reprendront dans les années 1930.

Notons que le thème du corporatisme est complètement absent des pages de L'Action française. La revue citait fréquemment des corporatistes français comme Georges Valois et René de La Tour du Pin, mais elle ne retenait de ces auteurs que leur critique du libéralisme et non leur projet de réforme sociale par l'organisation corporative. En fait, il faudra attendre les années de crise et la publication de l'encyclique Quadragesimo anno (1931) avant que les traditionalistes canadiens-français n'adhèrent au corporatisme social. Cette doctrine viendra compléter la pensée économique développée par Minville dans les années 1920, en lui donnant plus de cohérence. En fait, L'Action française pourrait être qualifiée de «quasi corporatiste» en ce sens qu'elle propageait la partie négative de l'idéologie corporatiste, soit l'antilibéralisme, mais ne préconisait pas encore sa partie positive, soit une organisation corporative de l'économie. Cependant, Minville avait déjà introduit dans la pensée de L'Action française l'idée du régionalisme économique que l'on retrouve également dans la doctrine du corporatisme social.

Le fil conducteur de la pensée d'Esdras Minville est le problème démographique. Son modèle d'économie régionale visait essentiellement à maintenir le poids démographique des Canadiens français. À cette époque, toute la société québécoise se préoccupait du problème de l'émigration vers les États-Unis, y compris le parti libéral. Mais à l'inverse du gouvernement Taschereau, Minville dénonçait avec

59. Voir François-Albert Angers, «La pensée économique d'Esdras Minville», AN, 65,910 (mai-juin 1976): 756-758. 
autant de vigueur l'exode rural vers Montréal que l'exode vers les États-Unis. Selon lui, les sources profondes de la nation canadiennefrançaise se trouvent à la campagne, sur le plan démographique comme sur le plan culturel ${ }^{60}$. Minville croit qu'un peuple ne peut pas survivre longtemps lorsque toute sa population est absorbée par la civilisation urbaine. D'une part, les populations rurales ont un taux de natalité supérieur et un taux de mortalité infantile inférieur à ceux des populations urbaines ${ }^{61}$. D'autre part, la ville déracine les traditions nationales. La vie urbaine, écrit Minville, tend à «mécaniser» l'individu; la spécialisation à outrance réduit les capacités de raisonnement; le progrès technique dispense de tout effort physique et mental; les loisirs artificiels, comme le cinéma, amenuisent l'intelligence et corrompent les mœurs. À l'inverse, la vie rurale constitue pour lui une sorte d'idéal social, moral et national.

Il ne faut pas percevoir la phobie urbaine de L'Action française comme un rejet de l'industrialisation. En fait, ce n'est pas la modernisation matérielle que la revue dénonçait mais bien l'américanisation sociale et culturelle. Notons d'abord que la phobie urbaine ne concernait que Montréal. La revue ne dénonçait jamais les villes canadiennes-françaises de taille moyenne ou petite comme Québec, Trois-Rivières et Chicoutimi. Si Montréal avait été une ville intégralement française, au lieu d'être une cité cosmopolite où prédominait la langue anglaise, l'attitude de L'Action française aurait pu être différente. Mais il fallait tenir compte de la réalité. De fait, l'ambiance de la métropole dans les années 1920 favorisait l'acculturation des Canadiens français.

Cependant, L'Action française n'entendait stopper ni le développement industriel ni même l'exode rural. Jean-Charles Magnan considérait l'émigration vers la ville comme «une loi économique à laquelle il faut se soumettre ${ }^{62} »$, et Charles Gagnier écrivait que «l'exode rural

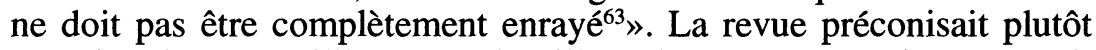
une situation d'équilibre entre la ville et la campagne qui permettrait aux Canadiens français de s'adapter graduellement au monde industriel tout en restant maîtres de leur vie économique et en préservant leurs traditions culturelles et religieuses. Elle proposait une modernisation à la canadienne-française axée sur le régionalisme économique et la petite industrie, plutôt qu'une modernisation à l'américaine fondée sur la concentration urbaine et industrielle.

60. Esdras Minville, «Le réservoir de la race», $A F$ (mai 1926): 258-276.

61. Dr Joseph Beaudoin, «La défense de notre capital humain. L'ennemi dans la place», AF (mars 1926): 130-146.

62. Jean-Charles Magnan, «L'émigration à la ville», $A F$ (avril 1926): 195-204.

63. Charles Gagnier, «Notre problème agricole (I). Les faits», $A F$ (février 1924): 91-104. 
L'Action française craignait surtout que le besoin d'émancipation économique des Canadiens français ne les amène à se départir de leur spécificité culturelle dans le but d'assimiler la mentalité anglosaxonne qui était, selon plusieurs, indissociable du succès matériel. En 1928, Beaudry Leman, gérant général de la Banque canadienne nationale et ancien collaborateur de L'Action française, prétendit que ses compatriotes devaient, pour relever le défi économique, renoncer à une partie de leur idéal français et adopter les «méthodes» qui faisaient le succès des Anglo-Saxons en affaires ${ }^{64}$. Or, selon L'Action française, le progrès économique n'exigeait pas une dénationalisation, même partielle, des Canadiens français. Minville fit remarquer à Leman que les Japonais et les Allemands avaient réussi à ébranler la suprématie commerciale des Britanniques et des Américains sans rien abandonner de leur identité nationale; qu'ils avaient, au contraire, «développé assez de virilité ethnique pour dompter les influences désagrégeantes et se hisser au premier rang ${ }^{65}{ }^{»}$. Il dénonçait vigoureusement le «funeste cliché» qui veut que les Canadiens français, parce que latins et catholiques, soient dépourvus du «sens des affaires». Selon Minville, il n'existe pas de méthodes commerciales anglosaxonnes; il n'y a que l'art du commerce, universel en ses principes, capable de s'adapter aux particularités culturelles de chaque peuple. Pour gagner la guerre économique, il n'est donc pas nécessaire de créer une civilisation hybride, mi-française et mi-américaine. Esdras Minville ne voit aucune antinomie entre le progrès économique et les principes traditionnels d'une civilisation française et catholique.

Nous touchons ici à l'objectif fondamental de la pensée économique d'Esdras Minville: concilier le progrès matériel avec la survie nationale et le salut spirituel des Canadiens français. Pour atteindre cet objectif, il conçut un modèle de développement alternatif axé sur la décentralisation régionale (équilibre ville-campagne) et économique (prédominance des PME) qui visait à contrer les problèmes causés par la concentration urbaine (le climat anglophone et irréligieux de Montréal) et industrielle (le «sur-État économique»).

\section{UN PROJET ALTERNATIF DE MODERNISATION}

Nous devons d'abord récuser les conclusions de Susan MannTrofimenkoff au sujet des trois caractéristiques de la pensée économique de L'Action française: 1) Le fait de lier la question économique à des préoccupations morales et de l'intégrer dans une

64. Beaudry Leman, «À travers notre correspondance (lettre à Lionel Groulx)», ACF (mai 1928): 288-293.

65. Esdras Minville, «L'éducation économique», ACF (mai 1928): 262-286. 
doctrine nationale ne constitue pas une approche «moraliste et doctrinaire», mais indique seulement que L'Action française voulait élaborer un projet de société global fondé sur les principes philosophiques et sociologiques du catholicisme. 2) Comme Antonio Perrault l'a bien démontré, toute entreprise économique ne repose pas nécessairement sur le matérialisme philosophique. C'est le libéralisme économique et non pas le progrès économique qui est implicitement matérialiste. 3) L'Action française ne croyait pas que l'industrialisation comportait plus de dangers que d'avantages. Au contraire, elle affirmait que le progrès matériel était indispensable pour assurer la survie nationale des Canadiens français et leur permettre de remplir leur «mission évangélisatrice» en Amérique du Nord. Dans son esprit, la révolution industrielle comportait à la fois un risque et un espoir. Les Canadiens français risquaient de perdre, dans ce bouleversement, leur identité latine pour devenir de simples «Saxons parlant français ${ }^{66}{ } »$; mais ils pouvaient également s'appuyer sur la conquête de la richesse matérielle pour devenir la «France d'Amérique», une nation rayonnante d'art, de culture, de science et de christianisme. L'Action française ne considérait pas l'industrialisation comme un écueil à éviter mais comme un défi à relever ${ }^{67}$.

Nous contestons également une des conclusions de la thèse de William F. Ryan qui affirme que si l'agriculturisme n'a exercé aucune influence concrète sur le processus d'industrialisation, il a quand même influencé l'idéologie des intellectuels traditionalistes. Or nous n'avons décelé aucune «dominante agriculturiste» dans la pensée de L'Action française, aucun «refus de l'âge industriel contemporain». Nous avons plutôt découvert un refus du libéralisme économique, le rejet d'une structure sociale particulière de l'ère industrielle contemporaine.

Dans une intéressante étude sur l'histoire de la pensée économique au $\mathrm{XIX}^{\mathrm{e}}$ siècle, Kevin Henley affirme que le nationalisme économique était une doctrine en soi, différente du libéralisme et du socialisme, mais qui était aussi répandue que ces deux dernières au Canada comme en Europe ${ }^{68}$. Selon lui, les principes du nationalisme

66. . Adélard Dugré, S.J., «Question de pédagogie. L’enseignement du français», $A F$ (août 1919): 353.

67. Patricia Dirks perçoit de la même façon que nous l'attitude des nationalistes devant les transformations économiques des années 1920. The Failure of L'Action libérale nationale (Montréal et Kingston, McGill-Queen's University Press, 1991), 13-17.

68. Kevin Henley, La doctrine du nationalisme économique au Canada central (18461885), thèse de doctorat (histoire), Université du Québec à Montréal, 1990, 408 p.; "La doctrine du nationalisme économique au Canada français (1846-1885)», Cahiers d'histoire, 10,2 (automne 1989): 32-53; «The International Roots of Economic Nationalist Ideology in Canada, 1846-1885», Journal of Canadian Studies/Revue d'études canadiennes, 24,4 (hiver 1989-1990): 107-121. 
économique sont les suivants: 1) l'économie politique fait partie de l'art du gouvernement et a pour but d'augmenter les forces productives de la nation; 2) le progrès économique doit être lié au progrès intellectuel et moral; 3) l'État représente la collectivité et doit intervenir pour parer à l'influence souvent néfaste de l'entreprise privée; 4) il faut un développement égal de toutes les nations et de tous les aspects de la vie économique: agriculture, industrie et commerce. Sur le plan pratique, les théoriciens du nationalisme économique sont en faveur d'un certain protectionnisme, de l'intervention de l'État, des progrès du syndicalisme et des lois sociales. Contrairement à l'école de Manchester, ils ont une approche pragmatique qui met l'accent sur l'industrie et l'agriculture plutôt que sur le commerce.

La doctrine du nationalisme économique était, avant l'émergence du socialisme, la principale force d'opposition aux thèses libérales. Ces principaux théoriciens furent Jean-Antoine Chaptal, Charles Dupin et Paul Cauwès en France, John Bernard Byle en GrandeBretagne, Henry C. Carey et Simon Patten aux États-Unis, Friedrich List et Eugen Dürhing en Allemagne. Le nationalisme économique, qu'il ne faut pas confondre avec l'autarcie, fut pratiqué par Bismarck en Allemagne, par John Quincy Adam aux États-Unis et par John A. Macdonald au Canada.

Les historiens connaissaient l'existence de cette doctrine mais la présentaient, soit comme une simple forme d'aide à la bourgeoisie nationale, donc une variante de l'économie libérale, soit comme une politique de compromis entre le libéralisme et le socialisme. L'originalité de la thèse de Henley est de considérer le nationalisme économique comme une doctrine en soi, aussi distincte du libéralisme que du socialisme. Il apporte ainsi un nouvel éclairage sur le débat idéologique qui a divisé le monde occidental au cours du XIX ${ }^{\mathrm{e}}$ siècle. Rompant avec la vision binaire de l'histoire des idéologies (libéralisme contre socialisme), il présente un débat qui met plutôt aux prises trois familles doctrinales ayant chacune un principe central différent: l'individu pour le libéralisme, la classe sociale pour le socialisme, la collectivité nationale pour le nationalisme. Selon Henley, la doctrine du nationalisme économique se rattache à la grande idéologie nationaliste occidentale, tout comme le clérico-nationalisme.

Discuter ici de la valeur intrinsèque de la thèse de Kevin Henley nous entraînerait trop loin de notre propos. Constatons seulement que les quatre énoncés qui caractérisent ce qu'il appelle la doctrine du nationalisme économique correspondent précisément à la pensée de L'Action française: 
1) La revue ne séparait pas l'économie politique des autres sciences sociales et morales; elle l'intégrait dans une doctrine de nationalisme intégral qui reposait sur la pensée sociale de l'Église. Son approche était empirique plutôt que théorique.

2) L'Action française rejetait l'individualisme libéral (l'idée du contrat social de Jean-Jacques Rousseau), et concevait la nation comme un tout organique supérieur à la somme de ses parties. Elle liait le progrès matériel au progrès moral et intellectuel.

3) La revue préconisait l'intervention de l'État pour corriger les effets néfastes du libéralisme économique sur les plans social (question ouvrière) et national (contrôle des ressources naturelles par les étrangers).

4) Le programme économique de L'Action française visait à promouvoir un développement égal de toutes les régions (équilibre ville-campagne) et de tous les secteurs d'activité (industrie et agriculture).

La pensée économique de L'Action française se rattacherait-elle à la doctrine du nationalisme économique telle que la définit Henley pour le XIX ${ }^{\mathrm{e}}$ siècle? C'est une hypothèse de recherche qu'il faudrait explorer. Henley n'a pas fait de lien entre le nationalisme économique du $\mathrm{XIX}^{\mathrm{e}}$ siècle et la doctrine sociale de l'Église du $\mathrm{XX}^{\mathrm{e}}$. Il semble pourtant $y$ avoir des points communs entre ces deux doctrines. Une étude d'ensemble de la pensée économique qui rejette à la fois le libéralisme et le socialisme, nous permettrait de mieux situer L'Action française dans le cadre des écoles doctrinales d'économie politique qui ont jalonné l'histoire intellectuelle des $\mathrm{XIX}^{\mathrm{e}}$ et $\mathrm{XX}^{\mathrm{e}}$ siècles. Or cette étude reste à faire.

Chose certaine, la doctrine économique de L'Action française ne se rattache pas à un quelconque «agriculturisme», à une idéologie spécifiquement canadienne-française qui aurait été, selon Michel Brunet, un sous-produit intellectuel du «repli agricole» provoqué par la Conquête. Elle se rattache plutôt à la doctrine sociale de l'Église, à un projet d' «économie chrétienne». Cette doctrine n'était ni rétrograde ni passéiste; elle était moderne et tournée vers l'avenir, mais elle envisageait cet avenir dans une optique différente du libéralisme et du socialisme. L'Action française proposait un projet alternatif de modernisation, un modèle de société qui accueillait les progrès scientifiques et économiques mais qui rejetait les valeurs libérales associées au modèle américain d'industrialisation.

Jusqu'à présent, l'originalité et la cohérence interne de ce projet alternatif de modernisation n'a pas été mis en lumière, parce que l'historiographie retient généralement une conception monolithique du 
progrès. Le développement économique et la modernisation de la société sont envisagés d'une seule façon, c'est-à-dire de la manière dont s'est effectivement modernisé le Québec, selon le modèle américain d'après la Seconde Guerre mondiale. Ainsi, le progrès devient nécessairement synonyme de grande industrie, de concentration urbaine, de disparition de la vie rurale, de laïcisation, d'éclatement des structures familiales traditionnelles, de matérialisme exacerbé et de contrôle par l'État de toute activité sociale. Cette conception unidimensionnelle de la modernisation repose sur une vision binaire des idéologies, comme si le progrès ne pouvait prendre que la forme du libéralisme ou du socialisme. Dès lors, toute autre idéologie est considérée comme étant en dehors de la «marche de l'histoire» et, par conséquent, «rétrograde» ou «passéiste».

Le concept de modernisation peut être entendu de deux manières. Il peut s'agir de la modernisation économique ou de la modernisation sociologique. La modernisation économique, qui n'est pas synonyme de concentration capitaliste, consiste à augmenter les forces productives par des améliorations techniques et scientifiques. La modernisation sociologique concerne plutôt le passage d'une société traditionnelle (la Folk Society des sociologues américains ${ }^{69}$ ) à des formes nouvelles d'organisation sociale (urbanisation, individualisme, laïcisation) qui sont généralement qualifiées de «modernes» mais qui ne sont, quant à nous, que «différentes». Or la modernisation économique n'est pas indissociable de la modernisation sociologique, bien qu'historiquement les deux phénomènes se soient produits simultanément. Du moins, L'Action française pensait qu'il était possible de moderniser l'économie québécoise sans bouleverser les structures sociales traditionnelles.

L'idéologie traditionaliste de L'Action française ne cadre pas avec la conception monolithique du progrès qui considère que la «modernisation» se ramène nécessairement à la modernisation sociologique, car la revue préconisait une autre forme de modernisation, un modèle de société qui visait, en quelque sorte, à «christianiser» la révolution industrielle. Pour ce faire, il fallait préserver les valeurs et les institutions traditionnelles comme la famille, la solidarité communautaire, la culture classique, l'ascendant social du clergé, le respect des hiérarchies et de l'autorité. Selon L'Action française, une économie décen-

69. Voir Everett C. Hugues, Rencontre de deux mondes. La crise d'industrialisation au Canada français (Montréal, Éditions Lucien Parizeau, 1944), 388 p.; Horace Miner, Saint-Denis: A French Canadian Parish (Chicago, University of Chicago Press, 1939), 299 p. Cette conception sociologique de la modernisation fut reprise par plusieurs auteurs dans Robert Comeau, dir., Économie québécoise. 
tralisée, demeurant à l'échelle humaine, permettait de réaliser ce projet de société plus facilement que le modèle américain d'économie libérale, un modèle qui provoquait inévitablement la concentration urbaine et le gigantisme industriel, la dépersonnalisation et l'atomisation sociale, le triomphe du matérialisme et la déchristianisation. Ce n'est pas parce que l'histoire s'est finalement déroulée d'une façon différente de ce qu'espérait L'Action française qu'il faut conclure que sa pensée «tournait le dos à l'avenir».

\section{CONCLUSION}

On peut s'interroger sur le réalisme du programme économique de L'Action française. Le Québec aurait-il pu fonder son économie sur la petite industrie et sur l'équilibre entre la ville et la campagne, alors que le reste de l'Amérique du Nord était balayé par la concentration urbaine et industrielle? Dans les années 1920, la classe financière anglo-montréalaise aurait-elle toléré une vigoureuse affirmation économique des Canadiens français appuyée par l'État provincial? Cependant, il faut noter que la relative émancipation économique du Québec actuel s'est en partie réalisée par les moyens que préconisait L'Action française, soit le progrès de l'instruction, la canalisation de l'épargne canadienne-française, les PME et la planification du développement par l'État. On peut reprocher à la revue d'avoir sousestimé l'importance des caisses populaires et de ne pas avoir envisagé un rôle plus actif de l'État dans le domaine financier. Mais d'une manière générale, son programme indiquait la bonne voie, surtout lorsqu'il liait la question économique au problème de la conscience nationale des Canadiens français.

L'histoire de la pensée économique au Québec n'en est qu'à ses débuts. Plusieurs pistes de recherche devront être explorées. Il faudrait identifier précisément les influences étrangères qui ont marqué l'œuvre de Minville et de Montpetit, faire une étude comparative avec la pensée économique des traditionalistes européens, analyser les points de convergence et les points de rupture entre les traditionalistes, les libéraux et les socialistes, tenir compte des nuances intermédiaires et situer l'évolution de la pensée économique dans une plus longue durée.

À notre avis, les futures recherches devraient surtout délaisser la vision binaire des idéologies et la conception unidimensionnelle du progrès, et tenir davantage compte du facteur religieux dans l'analyse des idéologies dites «de droite». Cela pourrait renouveler considérablement les études sur l'école de pensée traditionaliste. 